\title{
Descontaminação de Sinais de Eletrocardiograma via Wavelets
}

\author{
Henrique M. de Oliveira, Clodoaldo A. P. Lima e Marcio Eisencraft
}

\begin{abstract}
Resumo-Neste trabalho de iniciação científica são mostrados resultados computacionais do uso da descontaminação via wavelets de sinais de eletrocardiograma (ECG). Nas simulações foram utilizados sinais de ECG artificiais adicionados a ruído branco gaussiano. Compara-se o desempenho desta técnica utilizando diversas famílias de wavelets. Os resultados são promissores.
\end{abstract} ma.

Palavras-Chave- Descontaminação, wavelets, eletrocardiogra-

Abstract-In this short paper we show computational results of wavelet-based de-noising of electrocardiogram signals (ECG). It was used artificial ECG added to white gaussian noise. We compare the performance of this technique using different wavelet families. The results are auspicious.

Keywords—de-noising, wavelets, electrocardiogram.

\section{INTRODUÇÃO}

Neste trabalho estuda-se a decomposição de sinais de eletrocardiograma (ECG) por meio do método de wavelet shrinkage $[1,2]$.

Wavelets são um conjunto de funções-bases que atendem certos requisitos e são usadas para representar funções do tempo $f(t)$ [1]. Essas funções-base são construídas a partir de uma única função conhecida como wavelet-mãe $\psi(t)$ que deve atender as seguintes características [3]:

a) a área total sob a curva da função deve ser nula,

$$
\int_{-\infty}^{\infty} \psi(t) d t=0
$$

b) deve possuir energia finita,

$$
\int_{-\infty}^{\infty}|\psi(t)|^{2} d t<\infty
$$

Escolhida então uma wavelet-mãe, define-se um conjunto base de funções [2]

$$
\psi_{j, k}(t)=2^{j / 2} \psi\left(2^{j} t-k\right), \quad j, k \in \mathbb{Z}
$$

e a expansão wavelet de uma função $f(t)$ é dada por

$$
f(t)=\sum_{k=-\infty}^{\infty} \sum_{j=-\infty}^{\infty} a_{j, k} \psi_{j, k}(t) .
$$

Henrique M. de Oliveira, Clodoaldo A. P. Lima e Marcio Eisencraft, Escola de Engenharia, Universidade Presbiteriana Mackenzie, São Paulo, Brasil, e-mails: hen.oliveira@gmail.com,moraes@mackenzie.br, marcioft@mackenzie.br.
Os coeficientes wavelets $a_{j k}$ são obtidos pelo produto escalar entre $f(t)$ e $\psi_{j, k}(t)$.

\section{Descontaminação}

Uma das aplicações mais interessantes da transformada $w a-$ velets em Processamento Digital de Sinais é na descontaminação de sinais. Uma das técnicas desenvolvidas para isso é conhecida como wavelet shrinkage e foi elaborada por Donoho e Johnstone entre outros [4, 5].

Resumidamente, esta técnica consiste inicialmente em decompor o sinal por meio de transformadas wavelets. A seguir, em cada nível, eliminam-se os coeficientes de detalhe wavelets abaixo de um limiar fixo ou adaptativo. O limiar ainda pode ser rígido ou suave $[4,5]$. Por fim, o sinal é reconstruído a partir dos novos coeficientes wavelets.

O limiar a ser escolhido pode variar conforme o sinal, a $w a-$ velet e o ruído. Neste trabalho, utiliza-se um limiar fixo e rígido dado por [4 - 6]

$$
T_{m}=\frac{\sqrt{2 \log (k) \sigma}}{\sqrt{k}}
$$

em que $k$ é o comprimento da transformada no nível de decomposição $m$ e $\sigma^{2}$ é a variância do ruído.

\section{SIMULAÇÕES}

O sinal de ECG artificial utilizado aqui $s(t)$ é gerado por meio do algoritmo ecgsyn.m desenvolvido por P. McSharry e G. Clifford para o programa de simulação numérica Matlab ${ }^{\circledR}$ [7]. Utiliza-se uma freqüência de amostragem de $256 \mathrm{~Hz}$ e 60 batimentos por minuto. A esse sinal é somado um ruído branco gaussiano com média nula, gerando-se o sinal $s^{\prime}(t)$.

A eficiência do método é medida pelo ganho $G$ do sistema,

$$
G=\frac{S N R_{\text {out }}}{S N R_{\text {in }}}
$$

em que $S N R_{\text {in }}$ representa a razão entre a potência do sinal original e a potência do ruído branco gaussiano adicionado a ele e o valor de $S N R_{\text {out }}$ representa a razão sinal-ruído da saída, obtida pela razão entre a potência do sinal original e a potência do ruído do sinal recuperado, dado pela diferença entre o sinal original e o recuperado.

Utilizou-se uma decomposição em $m=2$ níveis. Esta escolha foi adotada por ser a que apresenta melhores resultados em testes preliminares.

Para a simulação foram utilizadas quatro diferentes famílias wavelets: (i) Haar (haar), (ii) Daubechies de ordem 9 (db9), 
(iii) Symlets de ordem 2 (sym2) e (iv) Coiflets de ordem 2 (coif2) [1].

Um exemplo de aplicação do método é mostrado na Figura 1. Em (a) mostra-se um trecho de sinal de ECG sem ruído e em (b) o mesmo trecho agora contaminado por ruído branco gaussiano com $S N R_{i n}=10 \mathrm{~dB}$. Na Figura 1(c) é apresentado o trecho de sinal recuperado utilizando a wavelet $\mathrm{db} 9$. Em (d) mostra-se o erro entre o sinal original e o contaminado e, por fim, em (e) o erro entre o sinal original e o recuperado. Neste caso, obteve-se uma $S N R_{\text {out }}=16.1 \mathrm{~dB}$ levando a um ganho de $G=6.1 \mathrm{~dB}$.
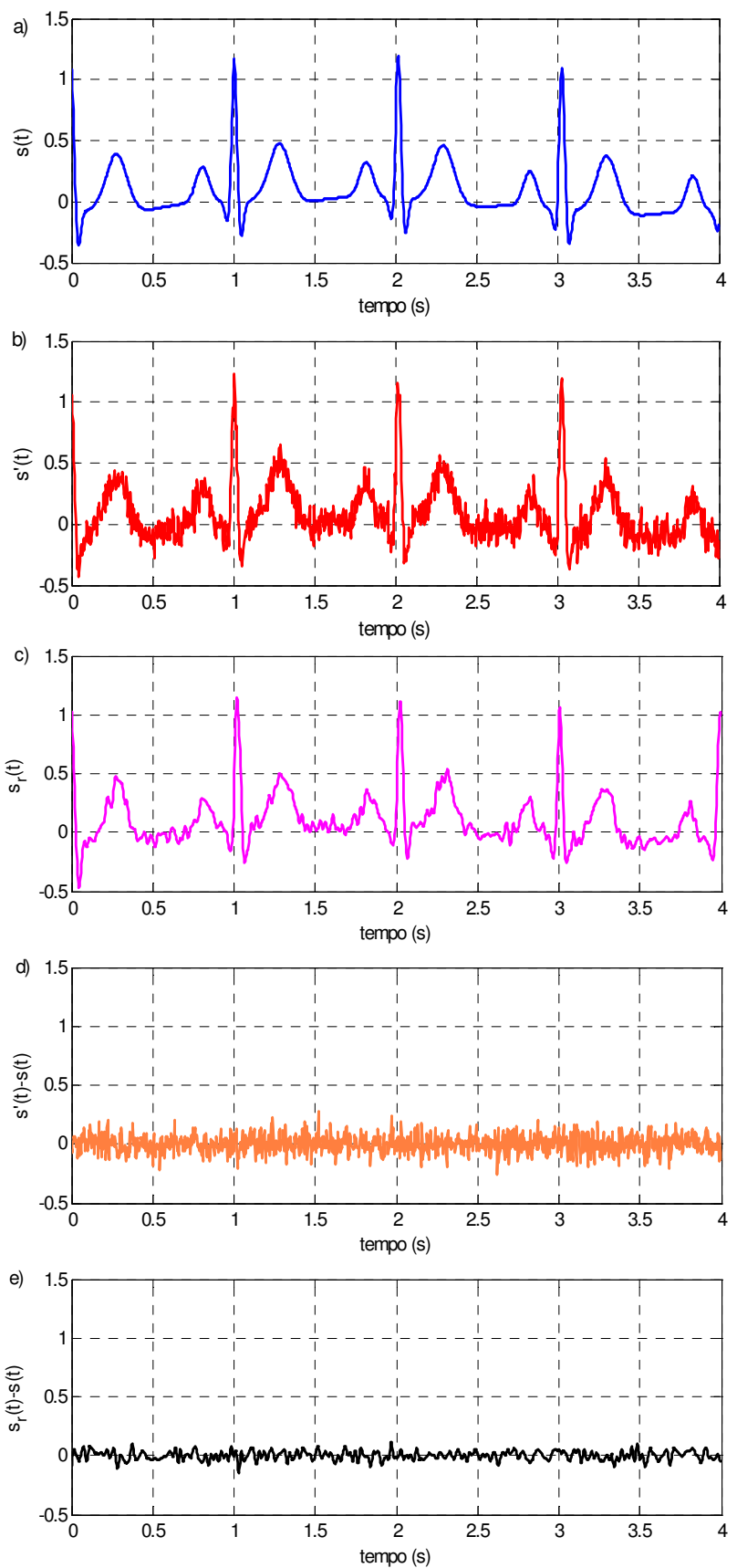

Fig.1. Sinais de eletrocardiograma: a) sinal original; b) Sinal contaminado; c) sinal recuperado $s_{r}(t)$; d) erro entre o sinal original e o contaminado; e) erro entre o sinal original e o recuperado.
A Figura 2 mostra uma comparação entre o ganho obtido para os quatro tipos de wavelets utilizadas em função da razão sinal ruído de entrada variando de -10 a $10 \mathrm{~dB}$.

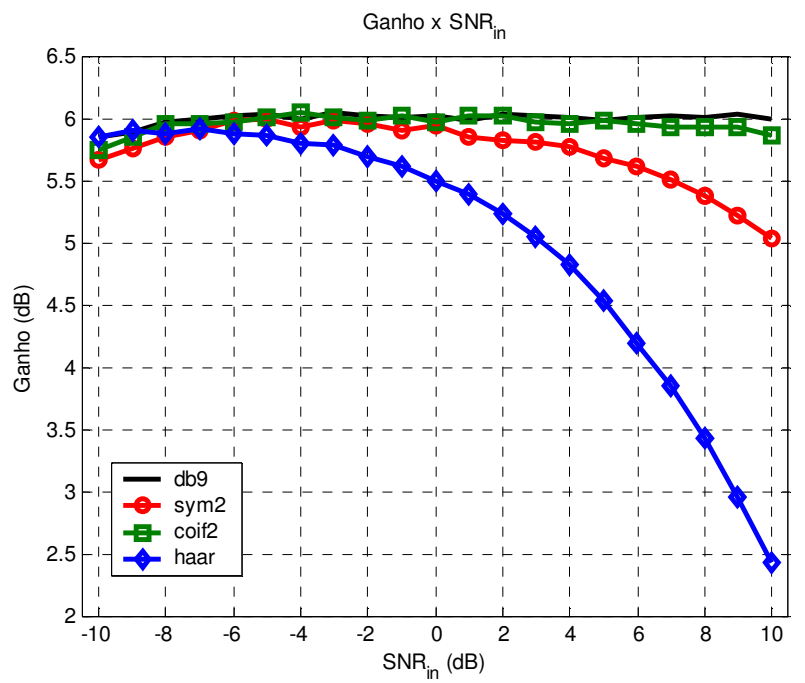

Fig.2. Comparação de ganho entre as wavelets utilizadas em relação à razão sinal-ruído de entrada

\section{CONCLUSÕES}

Este trabalho mostra alguns resultados referentes à aplicação do wavelet shrinkage para eliminação de ruído branco gaussiano de sinais de ECG. O método apresenta ganhos de aproxidamente $6 \mathrm{~dB}$ na razão sinal-ruído com o uso das wavelets coif2 e db9. Essas duas famílias apresentam superioridade as wavelets sym2 e haar a partir de $0 \mathrm{~dB}$, quando elas se mantêm constantes e as outras apresentam redução em seu ganho. A wavelet de haar é a que apresenta os menores ganhos para elevados níveis de razão sinal-ruído de entrada

Mais estudos devem ser feitos a fim de justificar teoricamente estes resultados.

\section{REFERÊNCIAS}

[1] G. Kaiser, A Friendly Guide to Wavelets, Birkhauser, 1994.

[2] S. Poornachandra, "Wavelet-based denoising using subband dependent threshold for ECG signals", Digital Signal Processing, n. 18, p. 49-55, 2008.

[3] S. Mallat, A Wavelet Tour of Signal Processing, Academic Press, 1999.

[4] H. M. de Oliveira, Análise de Sinais para Engenheiros Uma Abordagem via Wavelets. Brasport - Sociedade Brasileira de Telecomunicações, 2007.

[5] D.L. Donoho and I. M. Johnstone, "Threshold Selection for Wavelet Shrinkage of Noisy Data", Proceedings of the 16th Annual International Conference of the IEEE Engineering in Medicine and Biology Society, v.1, p. A24-A25, 1994.

[6] C. Taswell, "The What, How, and, Why of Wavelet Shrinkage Denoising", Computing in Science \&Engineering, v. 2, n. 3, p. 12-19, 2000.

[7] P. McSharry and G. Clifford, "A Dynamical Model for Generating Synthetic Electrocardiogram Signals", IEEE Transactions On Biomedical Engineering , v. 50, n. 3, p. $289-294,2003$. 